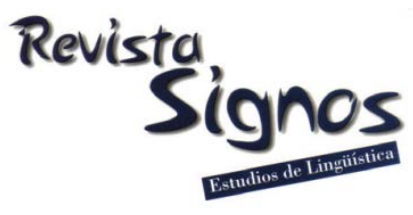

\title{
Complejidad, precisión y fluidez en el desempeño oral de aprendices con distintos niveles de proficiencia en inglés como L2
}

\section{Complexity, accuracy and fluency in oral performance of learners with different proficiency levels of English as L2}

\author{
J aviera Alfaro Chat Lilian Gómez Álvarez \\ UNIVERSIDAD DE CONCEPCIÓN \\ CHILE \\ UNIVERSIDAD DE CONCEPCIÓN \\ CHILE \\ ligomez@udec.cl \\ javalfaro@udec.cl
}

Katia Saez Carrillo

UNIVERSIDAD DE CONCEPCIÓN CHILE

ksaez@udec.cl

Recibido: 21-XII-2015 / Aceptado: 24-III-2017

\section{Resumen}

El desempeño oral en una segunda lengua (L2) se puede medir en términos de su complejidad, precisión y fluidez, otorgando una panorámica del aprendizaje de la L2. Según la perspectiva cognitiva, la teoría del procesamiento y el modelo de producción del habla, poseemos recursos atencionales limitados que dificultan la atención simultánea a las variables en estudio, a saber: Los constructos de complejidad, precisión y fluidez, y su relación con el desempeño oral en inglés como L2 en monólogos semiespontáneos en distintos niveles de proficiencia (A2, B2 y C1). La complejidad se midió en tanto diversidad léxica (como puntaje $\mathrm{D}$, parámetro válido y confiable para diversidad léxica) y complejidad gramatical (como promedio de extensión de palabras en cláusulas y número de cláusulas por unidad de Análisis del Habla (AH)). La precisión se midió como la proporción de unidades AH y cláusulas sin errores. Y la fluidez, como índice tiempo-fonación y promedio de duración de pausas. Todas las variables incrementaron a medida que se avanzaba en el nivel de competencia. Además, existe evidencia de interrelación de cada variable dentro de cada nivel. Las correlaciones significativas entre subcomponentes de un mismo constructo permiten sugerir una variable específica como representante del estado general de los distintos componentes del desempeño oral.

Palabras Clave: Complejidad, precisión, fluidez, niveles de proficiencia en inglés como L2, teorías cognitivas. 


\begin{abstract}
Oral performance in a second language (L2) can be measured in terms of complexity, accuracy and fluency, thus offering an overview of L2 learning. Focusing on the cognitive development of L2 learning, particularly from the point of view of Processing Theory and the Speech Production Model, limited attentional resources hinder simultaneous attention to the variables studied, namely: Performance components of complexity, accuracy and fluency in semi spontaneous oral monologues at different levels of English proficiency (A2, B2 and C1). Complexity was measured in terms of lexical range (as D score, best fit for lexical range) and grammatical complexity (as the average length of words in each clause, and number of clauses in each Analysis of Speech (AS) unit). Accuracy was measured as the proportion of AS units free from errors and percentage of error-free clauses. Fluency was measured by calculating the phonation-time ratio and the mean length of pauses. Regarding trends in L2 language development, results showed increase in all variables across all levels of proficiency. Besides, the variables studied are interconnected within each group sampled. Correlation analysis showed significant correlations between subcomponents within each construct, which suggests that any specific variable of each construct could represent the general state of the components of oral performance.
\end{abstract}

Key Words: Complexity, accuracy, fluency, proficiency levels of English as L2, cognitive theories.

\title{
INTRODUCCIÓN
}

Desde que la investigación en el campo de la adquisición de segundas lenguas (ASL) tomó un giro cognitivo (Pienemann, 1998; DeKeyser, 2015; Ullman, 2015), la triada complejidad, precisión y fluidez ha sido el foco de investigaciones (Skehan, 1998; Robinson, 2011; Larsen-Freeman, 2012; Vercellotti, 2012), emergiendo como epifenómeno de los procesos y mecanismos psicolingüísticos que subyacen a la adquisición, representación y procesamiento de los sistemas de una segunda lengua (L2) (Housen, Kuiken \& Vedder, 2012). Así, dichos constructos son componentes centrales del desempeño oral, de carácter multidimensional, por lo que, tanto las definiciones como la forma de medirlos abundan (Housen, Kuiken \& Vedder, 2012), dificultando comparar resultados (Ellis \& Barkhuizen, 2005).

En el presente estudio, se analiza el desarrollo del desempeño oral en inglés como L2 en tres niveles de proficiencia (A2, B2, C1), utilizando las medidas más recientes de análisis de los constructos de complejidad, precisión y fluidez, y buscando establecer relaciones entre ellos en los distintos niveles de competencia. Se espera así contribuir al estudio de la adquisición y el desarrollo de L2, mediante la observación de la interlengua de los discentes y la evaluación de supuestos teóricos existentes en la literatura cognitiva sobre el modelo de producción oral bilingüe, teoría de la atención y capacidad limitada, teorías de procesabilidad y automatización, y su conexión con las medidas de desempeño oral. 


\section{Marco teórico}

\subsection{Adquisición de Segundas Lenguas como ciencia cognitiva y desarrollo de L2}

Los enfoques cognitivos suelen basar sus hipótesis en la psicología cognitiva y la neurociencia, para comprender las representaciones mentales responsables de las funciones de alto orden (visión, lenguaje, categorización), viendo el proceso de adquisición de una L2 como la adquisición de una habilidad compleja, abordable al investigar cómo el cerebro humano procesa y aprende información nueva (ver, por ejemplo, DeKeyser, 2007).

Según la teoría de la procesabilidad (TP), en cualquier etapa del desarrollo de una L2, el aprendiente puede producir y comprender solo aquellas formas lingüísticas que el actual estado del procesador maneje. Por lo tanto, resulta clave entender la arquitectura del procesador y su forma de manejar la L2 para predecir el curso del desarrollo de las formas lingüísticas en la producción del lenguaje (Pienemann \& Lenzing, 2015). Por otro lado, la TP postula que durante el desarrollo de la L2 se acumulan reglas gramaticales y sus variantes, lo que permite desarrollar trayectorias de desarrollo de cada individuo mientras se adhieren al esquema general de desarrollo, explicando así el desarrollo universal de las etapas y de las variaciones individuales en cada una de ellas (Pienemann, 1998).

\subsection{Elementos que operan en el aprendizaje y producción de una L2}

Entre los elementos que operan en el aprendizaje y producción de L2, se encuentran: El conocimiento, el almacenamiento de la información, los recursos atencionales de los individuos, los procesos de automatización y de restructuración de la L2.

Para comenzar, el conocimiento de la L2 se representa como: Conocimiento declarativo o explícito, conocimiento de reglas y fragmentos de la lengua, y conocimiento implícito o procedimental, que es subconsciente y permite el uso rápido y efectivo de reglas y fragmentos de la comunicación, disponible automáticamente en producción espontánea.

En términos de almacenamiento de la información, se encuentran: La memoria de corto plazo (MCP), la memoria de largo plazo (MLP), y la memoria de trabajo (MT). La MCP es responsable de la activación temporal de la información lingüística. La MLP consiste en el vasto almacenamiento de conocimiento y registro de eventos previos. La MT extrae y almacena temporalmente información proveniente del input y de la MLP, que en aprendientes de L2 dependen en gran medida del procesamiento 
controlado más que del automático, dificultando el procesamiento para la comprensión y para la adquisición simultáneas (Ellis \& Barkhuizen, 2005).

Bajo el marco del procesamiento de la información, se concibe que la atención humana es una capacidad de naturaleza limitada (Leow \& Bowles, 2005), restringiendo la cantidad de atención que se puede prestar a la información. La atención se refiere a las funciones que regulan acciones y facilitan el aprendizaje sobre el ambiente, por ejemplo: La selección de información para procesamiento, activación de conceptos en la MLP y de participación coordinada en múltiples actividades simultáneas (Robinson, 2011).

Por otro lado, la automatización se refiere al control que se posee sobre el conocimiento lingüístico de la L2, es decir, cuán rápido, inconsciente y espontáneo (natural, sin esfuerzo) es el procesamiento de la L2, lo que DeKeyser (2007), en su teoría de adquisición de habilidades, concibe como conocimiento automatizado. En la misma línea, la restructuración alude a los cambios que sufren las representaciones internalizadas como consecuencia de un nuevo aprendizaje; estos son discontinuos y cualitativamente diferentes a aquellos en etapas previas. Así, aprender una L2 significa estructurar, organizar e integrar información nueva a la interlengua, mientras que un cambio en el sistema conduce a la reestructuración y reorganización de esta, creando un nuevo sistema lingüístico de la L2.

Finalmente, el desempeño de un aprendiz de L2 requiere de la ejecución de habilidades perceptuales, cognitivas y sociales que se facilitan por el uso rutinario (Gass, Behney \& Plonsky, 2013). Por consiguiente, convertir el conocimiento declarativo en procedimental es indispensable en la comunicación oral espontánea, ya que al acelerar los procesos de codificación léxica, gramatical y fonológica, se liberan recursos atencionales que pueden ser asignados al planeamiento del discurso, privilegiando las decisiones sobre el contenido del mensaje y el monitoreo, en vez de la precisión lingüística (Pawlak, 2011).

\subsection{El discurso oral en L2}

Con respecto al análisis del discurso oral, Foster, Tonkyn y Wigglesworth (2000) sugieren la unidad de Análisis del Habla (the analysis of speech unit / AS-unit): Un enunciado del hablante, compuesto por una cláusula independiente o unidad subclausal, junto con cualquier otra cláusula subordinada, asociada a cualquiera de las anteriores. En esta investigación, se hace referencia a dicha unidad de análisis como 'unidad AH'.

\subsubsection{Modelo de producción oral}

Un modelo lingüístico aplicado del habla debe explicar cómo y por qué los hablantes adaptan el conocimiento lingüístico al uso real de la lengua, evaluando si es apropiado en todos los niveles, a saber: Discursivo, léxico-gramatical y articulatorio. 
Este estudio se adscribe al modelo bilingüe de producción oral de Kormos (2006), que postula tres módulos en la producción del habla: Conceptualizador, formulador y articulador.

El procesamiento del habla en una L2 comienza con la conceptualización del mensaje e involucra la activación de conceptos relevantes que deben ser codificados, y la elección de la lengua del mensaje (L1 o L2). La mayoría de las emisiones orales son combinaciones de frases, cláusulas y oraciones memorizadas, esto es: El lenguaje formulaico. La codificación léxica se encarga de hacer coincidir las especificaciones conceptuales y el lenguaje de entrada con la entrada léxica apropiada. La siguiente fase es la codificación fonológica, que involucra la activación de la forma fonológica de la palabra codificada, y la silabación. El proceso final es el monitoreo, muy similar en L1 y L2.

Al igual que el modelo de Levelt (1989), el de Kormos asume la existencia de tres circuitos o momentos de monitoreo (monitoring loops): (1) Comparación del mensaje preverbal con las intenciones originales del hablante; (2) monitoreo del plan fonético (discurso interno) antes de la articulación; y (3) revisión del enunciado generado luego de la articulación. En cuanto se percibe un error o algo inapropiado en cualquiera de los momentos de monitoreo, se gatillan los mecanismos de producción por segunda vez. La diferencia más importante entre el monitoreo en la L1 y en la L2 es la utilización de los recursos atencionales disponibles, ya que, debido a que el procesamiento oral en una L2 requiere atención a nivel léxico, sintáctico y fonológico, se deben tomar decisiones conscientes sobre qué requiere más atención cuando se monitorea, lo que implica priorizar el contenido por sobre la forma, el léxico sobre la gramática, o viceversa (Kormos, 1999).

\subsubsection{Complejidad, precisión y fluidez como dimensiones del desempeño oral en L2}

Varios investigadores en el área de ASL (Ellis \& Barkhuizen, 2005; Kormos, 2006; Housen et al., 2012; Foster \& Skehan, 2012; Vercellotti, 2015) sostienen que la proficiencia en una L2 es de naturaleza multicomponencial, y que sus principales elementos pueden capturarse en las nociones de complejidad, fluidez y precisión.

La complejidad se entiende como lenguaje elaborado, que corresponde al límite máximo de la etapa de desarrollo actual de la interlengua del aprendiz (Ellis \& Barkhuizen, 2005), o bien a la etapa de reestructuración de la interlengua (Skehan \& Foster, 1997). Dicho de otro modo, es el uso de lenguaje más desafiante y difícil, con un repertorio más amplio de estructuras. Así mismo, la complejidad se considera como una función de sofisticación o una función de complejidad gramatical o sintáctica (Norris \& Ortega, 2009). 
La precisión se define como habla sin errores (Housen et al., 2012), es decir, que la estructura de la unidad de análisis del discurso oral coincida con la lengua meta. Por lo tanto, es importante determinar la variante con la que se contrasta la muestra. Para fines de la presente investigación, se consideró el inglés estándar (Standard English) como la norma.

El término fluidez se refiere a la manera de expresarse (delivery of speech) (Schmidt, 1992), y es de naturaleza multidimensional. Así, Skehan (2009), por ejemplo, distingue tres dimensiones: La velocidad (velocidad y densidad de las unidades lingüísticas producidas), desglose de la fluidez (número, longitud y ubicación de las pausas) y reparo de la fluidez (partidas falsas, errores de formulación, autocorrecciones, repeticiones).

\subsubsection{Relación entre complejidad, precisión y fluidez}

Como consecuencia de la capacidad limitada que poseen los humanos para procesar información y la restricción en el uso de los recursos atencionales, Skehan (1998) propone que, en la construcción del mensaje en L2, el aprendiz adulto enfatiza el significado sobre la forma, y que, cuando se focaliza en la forma, privilegia el control de esta (precisión) o el uso de elementos lingüísticos más avanzados (complejidad). Esto se conoce como 'efecto compensatorio' (trade-off), e implica que uno de los tres componentes del desempeño (precisión, fluidez o complejidad) se prioriza. Así, una tarea promueve solo fluidez, o complejidad y precisión (Robinson, 2011): En tareas monológicas simples, se enfatiza la fluidez, mientras que la precisión y la complejidad se enfatizan en tareas monológicas complejas. Skehan (2009) concluye que las tareas que implican información personal tienen mayor precisión y fluidez, y que la planificación pretarea permite producir emisiones más complejas y fluidas. En conclusión, se asume que los aprendices no pueden atender a todos los aspectos del desempeño de la lengua porque las demandas del procesamiento son mayores que su capacidad de atención, por lo que se prioriza la complejidad, precisión o fluidez en su desempeño, dependiendo del tipo de tarea (Ellis \& Barkhuizen, 2005).

\section{Marco metodológico}

Este estudio empleó un diseño no experimental de tipo transversal correlacional.

\subsection{Participantes}

Los participantes fueron 44 estudiantes de pedagogía en inglés de distintos años en su formación curricular, con nivel de inglés A2 (N=15), B2 (N=15) y C1 ( $=14)$, según el Marco Común Europeo de Referencia (Council of Europe, 2001). Fueron seleccionados y clasificados en un grupo de proficiencia según el puntaje obtenido en mock exams de las pruebas estandarizadas Preliminary English Test (PET), Fisrt Certificate in English (FCE), y Cambridge English Advanced (CAE), al finalizar cada semestre 
académico, evaluados por el docente a cargo de la asignatura de Lengua Inglesa. Los exámenes oficiales certifican el nivel de inglés de una persona, a saber: PET, A2; FCE, nivel B2; CAE, nivel C1.

\subsection{Materiales}

Para obtener el corpus oral, los tres grupos de estudio desarrollaron dos tareas de producción oral de un mock exam de la prueba TOEFL iBT, correspondientes a monólogos semiespontáneos. Estos fueron analizados y codificados con el fin de establecer la relación entre complejidad, precisión, fluidez, y el desempeño oral en distintos niveles.

\subsubsection{Tareas de producción oral del examen TOEFL iBT}

La prueba estandarizada de inglés Test of English as a Foreign Language (TOEFL iBT) es una evaluación multinivel (A1 a C1, MCER), de cuatro secciones: Comprensión auditiva, y lectora, producción oral, y escrita. En este estudio, se utilizaron las dos tareas independientes de la sección oral: El relato de una experiencia personal, y la elección justificada de una opción dada. En cada una, había 15 segundos de preparación, durante los cuales los aprendientes podían tomar notas y planear su respuesta, y 45 segundos para grabar la respuesta. Las mismas tareas fueron utilizadas con los tres grupos de la muestra.

\subsubsection{Transcripción y codificación}

Las tareas orales de cada sujeto se transcribieron y codificaron según los Códigos para el Análisis Humano de Transcripciones (Codes for the Human Analysis of Transcripts CHAT), en cláusulas y unidades $\mathrm{AH}$, forma óptima de dividir datos transcritos en unidades analizables (Foster et al., 2000; Vercellotti, 2015).

Posteriormente, las respuestas se analizaron utilizando CLAN (Computer Language Analysis) (MacWhinney, 2000), un software diseñado para analizar datos transcritos en formato CHAT. El lenguaje codificado se cuantificó usando comandos disponibles en el programa, como el promedio de palabras por unidad de habla y cláusulas. La codificación se realizó siguiendo la metodología de Foster et al. (2000), para identificar cláusulas y unidades $\mathrm{AH}$; en los siguientes ejemplos, se aprecia cómo las cláusulas sin errores se marcan con $[\wedge \mathrm{c}]$, las que presentan errores, con $\left[{ }^{\wedge} \mathrm{c}^{*}\right]$, y cada unidad $\mathrm{AH}$ está marcada por un punto (.) al final para indicar el límite.

Sujeto 04: It is important work in groups $\left[{ }^{\wedge} \mathrm{c}^{*}\right]$. (1 cláusula, 1 unidad $\mathrm{AH}$ ) Sujeto 29: The first one is communication $\left[{ }^{\wedge} \mathrm{c}\right]$ because when parents and their children communicate very well $\left[{ }^{\wedge} \mathrm{c}\right]$ they can solve problems easily $\left[{ }^{\wedge} \mathrm{c}\right]$. (3 cláusulas, 1 unidad $\mathrm{AH})$. 
En paralelo, se utilizó PRAAT (Boersma \& Weenink, 2015), para el etiquetaje del discurso en segmentos de habla (sounding) y pausas de silencio y rellenos (silent), todos aspectos relacionados con la medida de fluidez (ver Figura 1, como ejemplo).

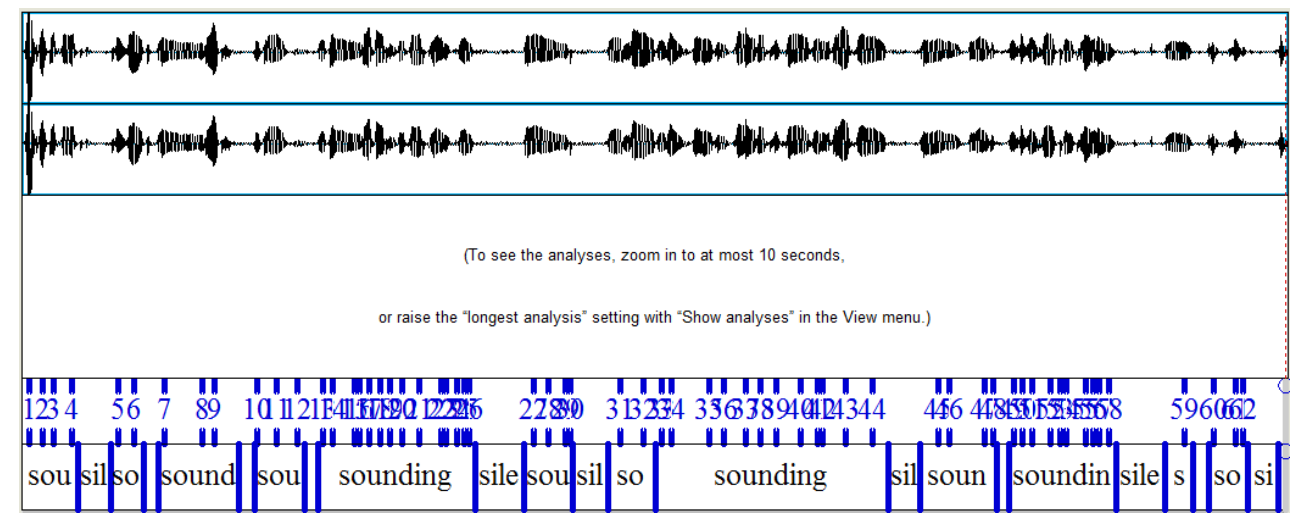

Figura 1. Ejemplo de etiquetaje en PRAAT, sujeto 17.

\subsection{Procedimiento}

Los discursos orales se grabaron en el laboratorio de lenguas en horario de clase. Cada aprendiente dispuso de un computador de escritorio y audífonos con micrófono incorporado. Se entrenó brevemente a los aprendientes para utilizar el software de la prueba TOEFL iBT (Educational Testing Service, 2012) y para grabar paralelamente sus respuestas en el computador. Luego, se procedió a tomar las muestras para la investigación.

\subsubsection{Análisis}

Debido a que los tres constructos del desempeño oral: Complejidad, precisión y fluidez, tiene múltiples subcomponentes, se necesitó más de una medida para cuantificarlos. La complejidad incluyó complejidad gramatical o sintáctica y diversidad léxica. En términos sintácticos, la complejidad se midió en tres niveles distintos: Complejidad global representada por el promedio de la extensión de la unidad AH, en otras palabras, por la 'longitud de la unidad AH' (Co1); la complejidad de frase, medida como 'longitud de la cláusula' (Co2) y complejidad por subordinación, que se calculó como el 'número de cláusulas dividido por las unidades AH' (Co3) (Norris \& Ortega, 2009; Vercellotti, 2015). Por otro lado, la diversidad léxica se calculó como valor D, utilizando el radio Tipo-Tipado (TTR) de las transcripciones en CHAT de manera automatizada, a través del comando vocd del programa CLAN, procedimiento que encuentra el mejor encaje entre la curva empírica y la curva teórica calculada del modelo, ajustando el valor del parámetro $\mathrm{D}$, que ha demostrado ser válido y confiable para diversidad léxica (McKee, Malvern, Richards, 2000; Vercellotti, 2015) sin los problemas de tamaño de muestras de otros métodos. 
El constructo precisión se midió a nivel global como 'porcentaje de unidades AH sin errores' (P1) y, a nivel de cláusula como 'porcentaje de cláusulas sin errores' (P2). Según Vercellotti (2012) y nuestra propia revisión bibliográfica, no existen investigaciones que analicen los efectos compensatorios en aspectos más específico de precisión, es decir, no se cuenta con evidencia de que un incremento en complejidad gramatical, variedad léxica, o fluidez afecte la precisión de alguna forma específica. En este estudio, al codificar una cláusula y unidad $\mathrm{AH}$ como correcta o errónea, se consideraron errores sintácticos y morfológicos; sin embargo, los errores de pronunciación no fueron considerados. Cuando ocurrió una autocorrección en el discurso, se tomó en cuenta solo la versión final.

La fluidez se midió utilizando el 'índice tiempo-fonación' (F1) y 'promedio de longitud de pausas' (F2), medidas que se han usado en estudios de de Jong y Perfetti (2011) y Vercellotti (2012). La variable F1 se calculó como tiempo hablado (excluyendo las pausas de relleno) dividido por tiempo total del habla. La longitud de las pausas se calculó como promedio de longitud de pausas de al menos 200 milisegundos, incluyendo pausas con elementos de relleno (e.g. ' $u$ ') y pausas de silencio. Se consideraron pausas con relleno tanto las pausas sonoras (er, erm, eh, ahm, uhm) como el alargamiento vocálico y consonántico, ya que el alargamiento segmental tiene una connotación pragmática, atribuible al planeamiento, ejecución, o la combinación de ambos. En términos de planeamiento, los alargamientos pueden producirse como mecanismo que permitan una fracción extra de tiempo para planear lo que se dirá (Cooper \& Paccia-Cooper, 1980).

Tabla 1. Resumen de las medidas aplicadas a cada discurso oral.

\begin{tabular}{|l|l|l|l|}
\hline \multicolumn{1}{|c|}{ Concepto } & \multicolumn{1}{|c|}{ Medición } & \multicolumn{1}{|c|}{ Definido como } & \multicolumn{1}{|c|}{$\begin{array}{c}\text { Tipo de } \\
\text { análisis }\end{array}$} \\
\hline Complejidad & Longitud de la unidad AH (Co1) & $\begin{array}{l}\text { Promedio del número de } \\
\text { palabras por unidad AH }\end{array}$ & Global \\
\hline Complejidad & Longitud de cláusula (Co2) & $\begin{array}{l}\text { Promedio de palabras por } \\
\text { cláusula }\end{array}$ & Subclausal \\
\hline Complejidad & Cláusulas/unidad AH (Co3) & $\begin{array}{l}\text { Proporción de cláusulas por } \\
\text { unidad AH }\end{array}$ & Subordinación \\
\hline Complejidad & Variedad léxica (Co4) & Puntaje D & Léxico \\
\hline Precisión & Precisión de unidad AH (P1) & $\begin{array}{l}\text { Proporción de unidades AH } \\
\text { sin errores }\end{array}$ & $\begin{array}{l}\text { A nivel de } \\
\text { unidad AH }\end{array}$ \\
\hline Precisión & Precisión de cláusula (P2) & $\begin{array}{l}\text { Proporción de cláusulas sin } \\
\text { errores }\end{array}$ & $\begin{array}{l}\text { A nivel de } \\
\text { cláusula }\end{array}$ \\
\hline Fluidez & Índice tiempo-fonación (F1) & $\begin{array}{l}\text { Tiempo de habla/ tiempo total } \\
\text { Global }\end{array}$ \\
\hline Fluidez & $\begin{array}{l}\text { Promedio de longitud de pausas } \\
\text { (F2) }\end{array}$ & $\begin{array}{l}\text { Promedio de longitud de } \\
\text { pausas (>200 ms) }\end{array}$ & $\begin{array}{l}\text { Desglose de } \\
\text { fluidez }\end{array}$ \\
\hline
\end{tabular}

Nota. Fuente: Adaptado de Vercellotti, M.L. (2012). Complexity, Accuracy, and Fluency as Properties of Language Performance: The Development of the Multiple Subsystems over Time and in Relation to Each Other (p.73). Tesis doctoral, Universidad de Pittsburg, Estados Unidos. 
Los resultados de las mediciones se analizaron con software estadísticos InfoStat v2015 y Rv3.2. Para determinar diferencias significativas en la producción oral de los tres grupos del estudio y los ocho subcomponentes de desempeño en el habla, se realizó un análisis de varianza (ANOVA), seguido del test de Tukey para comparaciones múltiples a posteriori. Para establecer relaciones entre los subcomponentes del habla se utilizó el coeficiente de correlación de Pearson. Para todos, se utilizó nivel de significancia de 0,05 .

\section{Resultados y discusión}

\subsection{Tendencias en el desarrollo de los constructos de complejidad, precisión y fluidez}

Se realizó un análisis de varianza (Tabla 2) para observar el comportamiento de los componentes complejidad, precisión y fluidez en cada grupo estudiado (A2, B2 y C1).

Tabla 2. Desempeño oral: Complejidad (C), Precisión (P) y Fluidez (F) por nivel de competencia en L2 (MCER).

\begin{tabular}{|l|c|c|c|c|c|c|c|}
\hline \multirow{2}{*}{ Variables } & \multicolumn{2}{|c|}{ Grupo A2 } & \multicolumn{2}{c|}{ Grupo B2 } & \multicolumn{2}{c|}{ Grupo C1 } & \multirow{2}{*}{ Valor p } \\
\cline { 2 - 7 } & Media (D.E.) & \multicolumn{2}{c|}{ Media (D.E.) } & \multicolumn{2}{c|}{ Media (D.E.) } & \\
\hline Co1 : Longitud de la unidad AH & $7,4(1,6)$ & $\mathrm{c}$ & $9,4(1,9)$ & $\mathrm{b}$ & $11,9(2,5)$ & $\mathrm{a}$ & $<0,0001$ \\
\hline Co2 : Longitud de cláusula & $6,2(1,1)$ & $\mathrm{b}$ & $7,2(0,9)$ & $\mathrm{a}$ & $7,8(0,8)$ & $\mathrm{a}$ & 0,0001 \\
\hline Co3 : Cláusulas/ unidad AH & $1,2(0,2)$ & $\mathrm{b}$ & $1,3(0,2)$ & $\mathrm{b}$ & $1,5(0,2)$ & $\mathrm{a}$ & 0,0005 \\
\hline Co4 : Diversidad léxica & $46,6(11,3)$ & $\mathrm{b}$ & $49,9(13,0)$ & $\mathrm{ab}$ & $58,4(9,5)$ & $\mathrm{a}$ & 0,0232 \\
\hline P1 : Precisión unidad AH & $37,2(14,4)$ & $\mathrm{b}$ & $53,6(20,1)$ & $\mathrm{a}$ & $61,5(14,9)$ & $\mathrm{a}$ & 0,0011 \\
\hline P2 : Precisión de cláusula & $43,9(12,2)$ & $\mathrm{b}$ & $59,8(22,0)$ & $\mathrm{a}$ & $72,5(13,0)$ & $\mathrm{a}$ & 0,0001 \\
\hline F1 : Índice tiempo fonación & $0,55(0,08)$ & $\mathrm{b}$ & $0,59(0,09)$ & $\mathrm{ab}$ & $0,65(0,06)$ & $\mathrm{a}$ & 0,0057 \\
\hline F2 : F2: Promedio longitud de pausas & $696,9(103,0)$ & & $735,8(201,5)$ & & $668,6(130,0)$ & & 0,4905 \\
\hline
\end{tabular}

Nota. D.E. $=$ desviación estándar; $\mathrm{p}>0,05$; Medias con una letra común no son significativamente diferentes (e.g. a, a / b,b); Medias con una letra distinta son significativamente distintas (a , b, c). Medidas con dos letras (ab) significa que no es significativamente distinta ni al valor acompañado por la letra $a$, ni al valor acompañado por $b$. Medidas sin letra no son estadísticamente significativas.

Los resultados obtenidos en la dimensión de complejidad evidencian una relación con el sistema subyacente de la L2. El un aumento progresivo de las variables Co1, $\mathrm{Co} 2, \mathrm{Co} 3$ y $\mathrm{Co} 4$ en los tres grupos en estudio parece vincularse con etapas de desarrollo del sistema subyacente de la L2. Por ejemplo, Housen et al. (2012) identifican la internalización de nuevos elementos de una L2 como una de las etapas de cambio: A medida que el aprendiente desarrolla su interlengua, esta se vuelve más elaborada y sofisticada. Se puede postular que cada grupo de estudio representa un estadio de la interlengua. Así, la producción de cláusulas más largas (Co2), de unidades AH más extensas (Co1), y de mayor número de cláusulas por unidad AH (Co3), asociada a la subordinación, puede entenderse como evidencia del incremento gradual de elaboración y complejidad de la interlengua.

Desde la perspectiva del modelo de producción oral de Kormos (2006), si entendemos el discurso oral como resultado del proceso de producción, los indicadores de complejidad (Co1, Co2, Co3 y Co4), utilizados para analizar el discurso 
en este estudio, constituirían evidencia de algunos de los procesos cognitivos subyacentes del módulo formulador, que es donde se producen las codificaciones léxico-gramaticales, morfo-fonológicas y fonéticas. Cada una de ellas está ligada a un tipo de conocimiento almacenado en la MLP. Al respecto, la medición de variables de complejidad deja entrever la posible relación entre el desarrollo de la interlengua y el conocimiento lingüístico del aprendiente sobre la L2: Su conocimiento declarativo y procedimental. Así, el aumento del número de cláusulas por unidad AH (Co3) podría revelar mayor conocimiento declarativo de las reglas de la L2, conocimiento implícito consistente en unidades de lenguaje formulaico y conocimiento inconsciente de reglas relativas a esquemas (e.g. suggest $+\mathrm{V}$-ing), y a esquemas mayores (e.g. construcción de cláusulas relativas) (Ellis \& Barkhuisen, 2005). A medida que mejora la competencia comunicativa en L2, el conocimiento declarativo se transforma en procedimental, lo que implica un aceleramiento en los procesos de codificación léxica, gramatical y fonológica (Pawlak, 2011), relacionado con el aumento de las variables Co3 y Co4 en el nivel C1 de competencia (C1) de este estudio.

Un fenómeno similar se observa en el desarrollo de los indicadores de precisión (P). Ambos subcomponentes de precisión (P1 y P2) mostraron incremento porcentual a medida que se avanzaba en el nivel de proficiencia. Esta tendencia podría indicar el desarrollo subyacente de la interlengua, en tanto cada etapa (grupo A2, B2 y C1) se caracteriza por la modificación del conocimiento de la L2, que implica procesos de reestructuración (McLaughlin, 1990) y sintonización del sistema de la L2 del aprendiente con la lengua meta (Housen et al., 2012); así, a medida que el aprendiente aumenta su experiencia en la L2 y aumenta su competencia comunicativa, hace un uso más preciso y complejo de la L2.

En contraste con lo anterior, y pese al incremento en ciertos valores de P1 y P2 en los tres grupos de estudio, no se encontraron diferencias estadísticamente significativas entre los promedios de P1 y P2 en los niveles de competencia B2 y C1, aunque estos valores fueron mayores que el promedio del nivel A2. En términos de desarrollo de la precisión, solo en el nivel A2 se observa un bajo porcentaje de construcciones correctas; luego se evidencia un progreso significativo cuando el aprendiente se encuentra en un nivel B2; sin embargo el incremento del constructo precisión se tiende a estabilizar entre los niveles de competencia comunicativa en inglés de nivel B2 y C1. Este fenómeno también puede tener explicación desde el punto de vista del desarrollo de la L2. De hecho, al hacer referencia al estudio longitudinal de Cancino, Rosansky y Schumann (1978), Ellis (1994) observa que, durante las primeras etapas, ocurren variaciones, que luego desaparecen, a medida que el aprendiente desarrolla una mejor organización del sistema en la L2.

En términos de fluidez, en todos los grupos de estudio se observó que el 'índice tiempo-fonación' (F1) fue aumentando a medida que se avanzaba en el nivel de 
competencia comunicativa en la L2. Es así como, de los 90 segundos destinados para la emisión oral, los aprendientes del nivel básico A2 hablaron 55\% del tiempo; los del nivel B2 59\%; y los del nivel C1 65\%. Esto podría evidenciar fenómenos subyacentes al proceso de producción oral, como el acceso automatizado al uso de unidades léxicas, por ejemplo. También podría relacionarse con la familiaridad temática de la tarea oral, lo que permitiría al aprendiz desarrollar, organizar y profundizar en el tema del que hablan.

En cuanto a la variable 'promedio de longitud de pausas' (F2), se esperaba que, a mayor nivel de proficiencia, disminuyera la longitud. Sin embargo, al contrastar el valor de F2 entre los grupos A2, B2 y C1, no existen diferencias estadísticamente significativas. De hecho, el habla del grupo C1 $(668,6)$ presentó un promedio de longitud de pausas menor, en contraste con los grupos A2 y B2, seguido por el grupo A2 $(696,9)$, mientras que, en el grupo B2, el comportamiento de esta variable fue inesperado, presentando el mayor promedio de longitud de pausas $(735,8)$. Este fenómeno podría estar ligado al intento por variar el vocabulario (medido como 'diversidad léxica', Co4), que podría requerir pausas más largas al no tener automatización de los procesos de formulación del mensaje, focalizando así la atención en la selección léxica y en la formulación del mensaje como tal.

En resumen, la disminución que se observó en el subcomponente 'índice de tiempo-fonación' (F1) y la ausencia de tal disminución en el 'promedio de longitud de las pausas' (F2) podrían guardar relación con el desarrollo de los sistemas complejos de una L2. Verspoor y Van Dijk (2014) postulan que la variabilidad implica puntos altos, regresiones, y momentos críticos que obedecen a distintos tipos de interacción entre los subsistemas de la interlengua. Dicha variabilidad también podría indicar diferencias individuales, como características de personalidad o estilo de habla (de Jong, Steinel, Florijn, Schoonen \& Hulstijn, 2012). Se observa una tendencia clara a aumentar la proporción habla/pausas, a medida que se avanza en la competencia en la L2, pese a que el promedio de longitud de pausas varía entre los distintos niveles. Entonces, el bajo porcentaje de pausas en el tiempo destinado a hablar deja entrever la existencia de procesos cognitivos subyacentes al procesamiento del habla, como la conversión del conocimiento declarativo en procedimental, que implica un aceleramiento en los procesos de codificación léxica, gramatical y fonológica (Pawlak, 2011), y que lleva finalmente a una automatización del sistema (Kormos, 2006). Lo anterior se puede traducir en aprendientes que al tener mayor nivel de proficiencia destinan menos tiempo a pensar, que hablan más, y que presentan emisiones más largas y complejas. Sin embargo, como esta investigación no profundizó en el contenido ni en el tipo de cláusulas utilizadas, no se puede aseverar si el discurso producido por el grupo $\mathrm{C}$, en cuanto al léxico utilizado y el tipo de construcciones sintácticas, está o no compuesto por elementos y estructuras que ya son familiares. 


\subsection{Análisis correlacional entre los subcomponentes de complejidad, precisión y fluidez}

Para cuantificar la relación de dependencia entre variables, se realizó un análisis correlacional (Tabla 3), utilizando el coeficiente de correlación de Pearson. Al observar los datos de la Tabla 3, se puede apreciar un patrón muy similar en el comportamiento de las correlaciones entre los tres niveles de competencia comunicativa en L2 (A2, B2 y C1) y los distintos subcomponentes de las variables de desempeño, a saber: Complejidad (C), precisión (P) y fluidez (F). Asimismo, se debe destacar que nueve de las 15 correlaciones fueron significativas a nivel 0,01. Finalmente, de las 27 correlaciones pareadas posibles, cuatro de ellas fueron significativas en los tres grupos de aprendientes:

1. Longitud de la unidad AH (Co1) con longitud de la cláusula (Co2)

2. Longitud de la unidad AH (Co1) con proporción clausula/unidad AH (Co3);

3. Precisión de la unidad AH (P1) con precisión de la cláusula (P2)

4. Índice de tiempo-fonación (F1) con promedio de longitud de las pausas (F2).

Tabla 3. Resumen de correlaciones entre los subcomponentes de complejidad (C), precisión

(P) y fluidez $(F)$ en los distintos niveles de inglés utilizando el coeficiente de correlación Pearson (r).

\begin{tabular}{|c|c|c|c|c|c|c|c|c|}
\hline Grupo A2 & Co1 & $\mathrm{Co} 2$ & $\mathrm{Co3}$ & Co4 & P1 & P2 & F1 & F2 \\
\hline Co1 : Longitud de la unidad AH & 1 &, $78^{* *}$ & ,59* & 0,08 & $-0,19$ & $-0,08$ & ,57* & $-0,37$ \\
\hline Co2: Longitud de cláusula & & 1 & $-0,03$ & $-0,09$ & $-0,14$ & $-0,24$ & 0,26 & $-0,13$ \\
\hline Co3: Cláusulas/unidad AH & & & 1 & 0,21 & $-0,09$ & 0,21 & ,55* & $-0,43$ \\
\hline Co4: Diversidad léxica & & & & 1 & 0,26 & 0,35 & 0,4 & 0,08 \\
\hline P1 : Precisión de unidad AH & & & & & 1 & ,87** & 0,25 & $-0,03$ \\
\hline P2: Precisión de cláusula & & & & & & 1 & 0,48 & $-0,33$ \\
\hline F1: Índice tiempo-fonación & & & & & & & 1 &,$- 54 *$ \\
\hline F2: Promedio longitud de pausas & & & & & & & & 1 \\
\hline Grupo B2 & Co1 & $\mathrm{Co} 2$ & Co3 & Co4 & P1 & P2 & F1 & F2 \\
\hline Co1 : Longitud de la unidad AH & 1 &, $73 * *$ &, $81 * *$ & $-0,27$ & 0,01 & 0,06 & 0,48 & 0,14 \\
\hline Co2: Longitud de cláusula & & 1 & 0,19 & $-0,25$ & $-0,06$ & $-0,1$ & 0,25 & 0,05 \\
\hline Co3: Cláusulas/unidad AH & & & 1 & $-0,14$ & 0,05 & 0,14 & 0,47 & 0,18 \\
\hline C4 : Diversidad léxica & & & & 1 & 0 & $-0,07$ & 0,12 & 0,07 \\
\hline P1 : Precisión de unidad AH & & & & & 1 & ,94** & 0,27 &,$- 57^{*}$ \\
\hline P2: Precisión de cláusula & & & & & & 1 & 0,26 & $-0,45$ \\
\hline F1 : Índice tiempo-fonación & & & & & & & 1 &,$- 57^{*}$ \\
\hline F2: Promedio longitud de pausas & & & & & & & & 1 \\
\hline Grupo C1 & Co1 & $\mathrm{Co} 2$ & Co3 & Co4 & P1 & P2 & F1 & F2 \\
\hline Co1 : Longitud de la unidad AH & 1 & $68^{* *}$ &, $88^{* *}$ & 0,33 & 0,17 & 0,35 & 0,38 & 0,06 \\
\hline Co2: Longitud de cláusula & & 1 & 0,25 & $-0,09$ & 0,11 & 0,24 & 0,26 & 0,22 \\
\hline Co3: Cláusulas/unidad AH & & & 1 & 0,48 & 0,14 & 0,29 & 0,35 & $-0,08$ \\
\hline Co4 : Diversidad léxica & & & & 1 & 0,15 & 0,07 & $-0,04$ & 0,12 \\
\hline P1 : Precisión de unidad AH & & & & & 1 &, $89 * *$ & 0,35 & $-0,06$ \\
\hline P2: Precisión de cláusula & & & & & & 1 & 0,48 & $-0,22$ \\
\hline F1 : Índice tiempo-fonación & & & & & & & 1 &,$- 71 * *$ \\
\hline F2: Promedio longitud de pausas & & & & & & & & 1 \\
\hline
\end{tabular}

Nota. ${ }^{* *}$ Correlación significativa a nivel 0,01 (bilateral). ${ }^{*}$ Correlación significativa a nivel 0,05 (bilateral). 
Se observó que la variable 'longitud de la unidad AH' (Co1) correlaciona altamente con la variable 'longitud de cláusula' (Co2), con un índice de Pearson de 0,78 para el grupo A2, 0,73, para el B2, y 0,68, para el nivel C1 de competencia; esto es: La extensión de la unidad $\mathrm{AH}$ en el discurso oral de un aprendiente de cualquiera de estos tres niveles de inglés mostró correlación positiva con la extensión de la cláusula, es decir, cuando el promedio de la cantidad de palabras que conformaban una unidad AH aumentó, también lo hizo el promedio de la cantidad de palabras de una cláusula. Vercellotti (2015) también encontró correlación positiva entre los subcomponentes de complejidad Co2 y Co1.

Por otra parte, el factor Co1 muestra correlaciona positiva de 0,59, 0,81 y 0,88 en los grupos A2, B2 y C1, respectivamente, con la variable Co3; esto es: Cuando el promedio de la cantidad de palabras de una Unidad AH (Co1) aumentó, también incrementó la cantidad de cláusulas en una unidad AH (Co3) y viceversa. Vercellotti (2012) también concluye que ambas variables tienen un alto grado de correlación a nivel del desarrollo del discurso oral en un individuo $(r=.8365)$ así como también entre individuos $(r=.8365)$. Además, es lógico que el incremento de la proporción de cláusulas por Unidad AH (Co3) vaya de la mano con un aumento en la cantidad de palabras que conforman una unidad AH (Co1).

Con respecto a la correlación entre las variables $\mathrm{Co} 2$ y $\mathrm{Co} 3$, el resultado no fue estadísticamente significativo en ninguno de los grupos. En una investigación previa longitudinal (Vercellotti, 2012) se reportó una correlación negativa entre las variables Co2 y Co3, lo que sugeriría que un incremento en el número de cláusulas disminuiría su longitud; sin embargo, en dicho estudio, el análisis de las variables de complejidad se realizó individualmente, a diferencia del presente estudio, en que se concentraron los datos de todos los aprendientes como un solo grupo; de hecho, al realizar la misma correlación entre individuos (a nivel grupal), Vercellotti (2012) informa que la correlación negativa entre las variables no se expresó, lo que coincide con los resultados de la presente investigación. En resumen, considerando que el nivel de análisis (intra o inter individuo) de las correlaciones de los subcomponentes Co2 y Co3 puede explicar la diferencia en las correlaciones, se podría pensar que las correlaciones del presente estudio representarían lo que sucede a nivel grupal (entre individuos), que no es evidente a nivel individual.

Si bien, resta confirmar dicha hipótesis en un futuro estudio, no se puede descartar que los resultados se explicaran por la variabilidad del desarrollo individual. Según la teoría de la procesabilidad (TP) (Pienemann, 1998), cada etapa representa un conjunto de reglas gramaticales que comparten determinadas rutinas de procesamiento; al ir construyendo su interlengua y avanzando en la adquisición de su L2, los aprendientes acumulan reglas gramaticales y sus variantes, que les permiten desarrollar trayectorias de desarrollo individuales mientras se adhieren al esquema general de desarrollo. Así, 
la TP explica el avance universal de desarrollo de una L2 y de las variaciones individuales en cada etapa.

En general, las variables 'longitud de cláusula' (Co2) y 'proporción cláusula/unidad AH' (Co3) podrían explicar el aumento de la 'longitud de la unidad AH' (Co1) (Tabla 2). $\mathrm{Y}$, aunque intuitivamente se esperaría que el aumento de $\mathrm{Co} 2$ redundara en aumento de la cantidad de cláusulas de una unidad $\mathrm{AH}$ (Co3), esto no fue refrendado por lo datos de este estudio: No hubo correlación entre Co2 y Co3 en ninguno de los niveles de competencia.

En cuanto al componente de precisión, se observó una alta correlación ( $\mathrm{p}>$ 0,01: 0,87 para el grupo A2 de competencia; 0,94 para el B2, y 0,89 para el C1) entre los subcomponentes 'precisión unidad AH' (P1) y 'precisión cláusula' (P2) (Tabla 3); así, se podría concluir que, al aumentar la cantidad de unidades AH libre de errores, también aumenta la precisión a nivel de cláusula. Similares resultados se advierten en otros estudios (e.g. Vercellotti, 2012) al analizar el discurso individual de estudiantes de inglés como L2, con correlación alta en las medidas de precisión (correlación individual $r=0,876$, y entre individuos, $r=0,700)$. En consecuencia, este estudio adhiere a la sugerencia de Vercellotti (2012) de que, en futuras investigaciones solo se utilice una de las medidas: 'Precisión unidad AH' (P1) o 'precisión de cláusula' (P2), dependiendo del nivel de análisis.

Finalmente, respecto de los subcomponentes de fluidez F1 y F2, se observó una correlación negativa en los tres niveles: A2, B2 y C1 (-0,54, -0,57 y -0,71, respectivamente); es decir, al aumentar la longitud de las pausas, disminuyó el índice tiempo-fonación, y viceversa. Estos resultados concuerdan con los de Vercellotti (2015) y se condicen con la evidencia estadística del ANOVA del presente estudio, que muestra que, tanto en el grupo A2 como en el C1, la variable F1 fue mayor que la F2; esto es: A mayor tiempo de habla, hubo disminución en la duración de las pausas, lo que es coherente con la diferencia significativa en los valores de F1 entre los polos (nivel A2 y C1). Cabe recordar que, en el ANOVA, el grupo B2 no mostró el mismo comportamiento que los niveles A2 y C1, en la relación entre las variables F1 y F2; sin embargo, en el análisis correlacional, sí hubo relación estadísticamente significativa ( $p$ $<0,05)$. Al considerar la DS de la variable 'longitud de pausas' del nivel B2 $(201,5)$, y analizar los datos individuales del grupo, se observa que de los 15 aprendices, tres mostraron una alta dispersión, lo que podría explicar los resultados del ANOVA y reforzaría lo postulado por de Jong et al. (2012) sobre ciertos elementos que pueden influir en las medidas de fluidez, como, por ejemplo, las diferencias individuales, tales como las características de personalidad o estilo personal de habla.

De acuerdo a la literatura revisada, se esperaba encontrar efectos compensatorios (trade-off) entre complejidad, precisión y fluidez en los distintos niveles de proficiencia, basándose en la teoría de la capacidad y la limitación atencional del aprendiente de una 
L2, que lo obligaría a priorizar un área (complejidad, precisión o fluidez) de desempeño sobre las otras; sin embargo, en este estudio, los componentes de complejidad, precisión y fluidez no revelaron una relación compensatoria en el desempeño oral de los aprendientes. La existencia de una correlación positiva entre las variables complejidad gramatical y fluidez se postuló siguiendo a Skehan (2009), quien indica que la planificación pretarea facilita la producción de emisiones más complejas y fluidas. Los datos de este estudio validan tal hipótesis para el nivel A2, pero no para los niveles B2 ni C1. La correlación positiva entre los subcomponentes de complejidad 'longitud de la unidad AH' (Co1) y 'proporción cláusulas/unidad AH' (Co3) en el nivel A2 sugiere que las unidades $\mathrm{AH}$ equivalen a una cláusula; asimismo, era esperable la correlación positiva entre 'proporción cláusulas/unidad AH' (Co3) e ‘índice tiempo-fonación' (F1), ya que la producción de una cláusula equivale al tiempo de una emisión oral sin pausas mayores a 200 milisegundos. Lo anterior es evidencia del limitado uso de la L2 que tienen los aprendices de nivel A2 y de la complejidad sintáctica de sus emisiones, característica propia de la competencia lingüística en esta etapa de interlengua. Otra posible explicación para la ausencia de trade-off radica en variables ajenas a este estudio, como ciertas características de personalidad (e.g. Dewaele \& Furnham, 1999, 2000). Al trabajar con sujetos extrovertidos en su capacidad de procesamiento paralelo, Eysenck (1981) observó que dicho procesamiento podría permitir mantener la fluidez a la vez que el hablante de L2 complejiza y monitorea su discurso.

En segundo lugar, basándose en el límite de capacidad atencional (Kormos, 1999; Skehan, 2009), se esperaba obtener una correlación negativa entre las medidas de fluidez y precisión en el nivel A2, de modo que el aprendiente priorizara el contenido (fluidez) por sobre la forma (precisión), o viceversa. Además, siguiendo la teoría de la automatización, se esperaba que una mayor cantidad de unidades $\mathrm{AH}$ y de cláusulas libres de errores (precisión) implicara pausas más largas y un 'índice tiempo-fonación' menor. Aunque no se observó correlación entre estas variables en los grupos A2 y C1, se encontró correlación negativa entre fluidez (F2: Promedio longitud de las pausas) y precisión (a nivel de unidad AH) en el grupo B2; en otras palabras, los aprendientes de nivel intermedio tendieron a producir más unidades $\mathrm{AH}$ sin errores cuando las pausas de su discurso oral tenían menor duración, lo que contrasta con lo predicho por Skehan $(1998,2009)$ sobre la relación compensatoria que existe entre el significado (fluidez) y la forma (precisión). Dicha correlación negativa se puede explicar, por un lado, por el uso de estructuras y vocabulario familiar, que supone procesos de acceso léxico más automatizado, permitiendo a los aprendientes acceder rápidamente a la información almacenada. En contraste, pausas largas pueden ser evidencia del esfuerzo por elaborar construcciones (a nivel sintáctico y léxico) más complejas, con el consecuente efecto negativo en la unidad $\mathrm{AH}$, debido a los errores. 


\subsubsection{Casos particulares de correlación significativa entre variables}

En tanto fluidez, el ‘índice tiempo fonación’ (F1) correlacionó positivamente con los subcomponentes de complejidad: 'Longitud de la unidad AH' (Co1) y 'proporción cláusulas/unidad AH' (Co3) en el nivel A2. Tales correlaciones implican que, al aumentar la extensión de la unidad AH, también aumentó la duración del tiempo del habla; además, cuando la proporción de cláusulas contenidas en una unidad $\mathrm{AH}$ aumentó o disminuyó, el tiempo de habla también lo hizo en la misma dirección. Lo anterior sugiere que la mayoría de los enunciados emitidos correspondió a una unidad AH (nivel sintáctico) y, al obtener una correlación positiva en la proporción cláusula/unidad $\mathrm{AH}$, se refuerza la observación de que las unidades $\mathrm{AH}$ de los aprendientes de nivel A2 equivalen a una cláusula, fenómeno que no se observa en el habla de los aprendices de los niveles superiores: B2 ni C1.

Otra correlación que se dio solo en uno de los niveles de competencia corresponde a la correlación negativa $(-0,57)$ entre las variables 'precisión de la unidad AH' (P1) y 'promedio de longitud de las pausas' (F2), para el nivel B2 de competencia. Así, cuando los aprendientes de dicho nivel produjeron un alto número de unidades $\mathrm{AH}$ sin errores (P1), el promedio de duración de las pausas (F2) en su discurso oral disminuyó. Esta correlación negativa se puede explicar por la probabilidad de que, a nivel sintáctico y léxico, la lengua utilizada en el discurso estuvo compuesta por estructuras y vocabulario familiar, por lo tanto, como estas secuencias de elementos se automatizan como rutinas neuromotoras (Beckner, Blythe, Bybee, Christiansen, Croft, Ellis, Holland, Ke, Larsen-Freeman \& Schonemann, 2009), la planificación y ejecución del discurso son también automáticas; es decir, los aprendientes pudieron acceder rápidamente a la información almacenada. En contraste, largas pausas se relacionan con un intento por realizar construcciones más complejas (a nivel sintáctico y léxico), al borde de su nivel de desarrollo, aumentando las posibilidades de cometer errores, que impactarán la unidad $\mathrm{AH}$, aunque fueran menores.

\section{CONCLUSIONES}

Los resultados de esta investigación permiten caracterizar el desempeño oral de aprendientes de inglés como L2 en situación de instrucción en un programa de pseudoinmersión, con una tendencia de incremento en cada medida de los constructos estudiados: Complejidad, precisión y fluidez, ya que el valor de cada variable aumentó a medida que se avanzaba en el nivel de competencia comunicativa en inglés como L2.

Cabe destacar las correlaciones significativas entre algunos subcomponentes de dichos constructos, lo que permite sugerir lo siguiente: (1) Para medir la complejidad gramatical de un discurso oral a nivel global, se necesitaría medir solo la variable 'promedio longitud de unidad AH' (Co1); (2) para medir la complejidad léxica, se propone utilizar la medida 'diversidad léxica' (Co4), puesto que la ausencia de 
correlación entre Co4 y las variables de complejidad gramatical sugiere que la complejidad gramatical y léxica no pueden medirse con un único índice; (3) la correlación significativa entre las medidas 'precisión de la unidad AH' (P1) y 'precisión de la cláusula' (P2) indica que, dependiendo del nivel de análisis de la investigación (global o a nivel de cláusula), se podría medir solo la 'precisión de la unidad AH' (P1) o solo la 'precisión de la cláusula' (P2), como variable representativa del estado de la precisión en el discurso oral.

Por su parte, la correlación negativa significativa que demostraron las variables del constructo fluidez (F1 y F2) implica que, dependiendo del foco de estudio de la investigación, se podría medir solo el 'índice tiempo-fonación' (F1) como representante del subsistema fluidez, si el interés del estudio recae en cuantificar el tiempo de producción del aprendiz, y no se busca profundizar en las pausas ni en su significado.

En resumen, los resultados obtenidos constituyen un aporte al campo de la ASL por su uniformidad en el desarrollo lineal de la mayoría de las medidas, desestimando los efectos compensatorios en el desarrollo de la competencia en inglés como L2. En cuanto a las correlaciones, los resultados refuerzan la fuerte relación entre los subcomponentes de un mismo constructo entre Co1-Co2 y Co1-Co3, P1-P2, F1-F2.

Finalmente, aunque los datos cuantificables del desempeño oral obtenidos en esta investigación parecen no indicar un desarrollo significativo entre niveles adyacentes de proficiencia en inglés como L2, es posible que se pueda encontrar evidencia más robusta de esta progresión utilizando una metodología de trabajo complementaria, por ejemplo, la propuesta por Larsen-Freeman (2012). Se sugiere, entonces, continuar realizando investigaciones que analicen el desempeño oral en L2 desde la teoría de la complejidad, lo cual implicaría adoptar una perspectiva de sistema, más allá de las relaciones no lineales, causa-efecto, entre variables estudiadas por separado. Sería deseable realizar observaciones a nivel individual, para ver cómo se comportan las medidas utilizadas en el presente estudio en cada aprendiente, y luego agruparlos por nivel para ver si se puede identificar algún patrón en cada grupo o nivel de competencia. Con todo, el presente esfuerzo por aplicar la teoría de la complejidad a fenómenos de ASL es en sí una contribución a un área que recién está despegando y que tiene gran potencial para develar respuestas a preguntas pendientes sobre los procesos y los mecanismos psicolingüísticos que subyacen a la adquisición, representación y procesamiento de los sistemas de una L2.

\section{REFERENCIAS BIBLIOGRÁFICAS}

Beckner, C., Blythe, R., Bybee, J., Christiansen, M., Croft, W., Ellis, N. C., Holland, J., Ke, J., Larsen-Freeman, D. \& Schonemann, T. (2009). Language is a complex adaptive system: Position paper. Language Learning, 59(1), 1-27. 
Boersma, P. \& Weenink, D. (2015). Praat: Doing phonetics by computer. Versión 6.0.08 [en línea]. Disponible en: http://www.praat.org/

Cancino, H., Rosansky, E. \& Schumann, J. (1978). The acquisition of English negatives and interrogatives. En E. Hatch (Ed.), Second Language Acquisition: A book of readings (pp. 207-230). Rowley, MA: Newbury.

Cooper, W. \& Paccia-Cooper, J. (1980). Syntax and speech. Cambridge: Harvard University Press.

Council of Europe. (2001). Common European Framework of Reference for Languages: Learning, teaching, assessment. Cambridge: Cambridge University Press.

de Jong, N. \& Perfetti, C. (2011). Fluency training in the ESL classroom: An experimental study of fluency development and proceduralization. Language Learning, 61(2), 1-36.

de Jong, N., Steinel, M. P., Florijn, A., Schoonen, R. \& Hulstijn, J. H. (2012). Linguistic skills and speaking fluency in a second language. Applied Psycholinguistics. Advance online publication, 34(5), 893-916.

DeKeyser, R. (2007). Skill acquisition theory. En B. VanPatten \& J. Williams (Eds.), Theories in second language acquisition: An introduction (pp. 97-113). Nueva Jersey: Lawrence Erlbaum.

DeKeyser, R. (2015). Skill acquisition theory. En B. VanPatten \& J. Williams (Eds.), Theories in Second Language Acquisition: An Introduction (pp. 94-112). Nueva York: Routledge.

Dewaele, J. M. \& Furnham, A. (1999). Extraversion: The unloved variable in Applied Linguistics research. Language Learning, 49(3), 509-544.

Dewaele, J. M. \& Furnham, A. (2000). Personality and speech production: A pilot study of second language learners. Personality and Individual Differences, 28(2), 355-365.

Educational Testing Service. (2012). The official guide to the TOEFL iBT test. (4th Ed.). Nueva Jersey: McGraw-Hill.

Ellis, R. (1994). The study of second language acquisition. Oxford: Oxford University Press.

Ellis, R. \& Barkhuizen, G. (2005). Analysing learner language. Oxford: Oxford University Press.

Eysenck, H. (1981). General features of the model. En H. Eysenck (Ed.), A model for personality (pp. 1-37). Berlin: Springer. 
Foster, P. \& Skehan, P. (2012). Complexity, accuracy, fluency and lexis in task-based performance: A synthesis of the Ealing research. En A. Housen, F. Kuiken \& I. Vedder (Eds.), Dimensions of L2 performance and proficiency: Complexity, accuracy and fluency in SLA (pp. 199-220). Amsterdam: John Benjamins.

Foster, P., Tonkyn, A. \& Wigglesworth, G. (2000). Measuring spoken language: A unit for all reasons. Applied Linguistics, 21(3), 354-375.

Gass, S., Behney, J. \& Plonsky, L. (2013). Second language acquisition: An introductory course. Nueva York: Routledge.

Housen, A., Kuiken, F. \& Vedder, I. (2012). Dimensions of L2 performance and proficiency: Complexity, accuracy and fluency in SLA. Nueva York: John Benjamins.

Kormos, J. (1999). Monitoring and self-repair in L2. Language learning, 49(2), 303-342.

Kormos, J. (2006). Speech production and second language acquisition. Magwag, Nueva Jersey: Lawrence Erlbaum.

Larsen-Freeman, D. (2012). Complexity theory. En S. Gass \& A. Mackay (Eds.), The Routledge handbook of second language acquisition (pp. 73-87). Nueva York: Routledge.

Leow, R. \& Bowles, M. (2005). Attention and awareness in SLA. En C. Sanz (Ed.), Mind and context in adult second language acquisition: Methods, theory and practice (pp. 179-203). Georgetown: Georgetown University Press.

Levelt, W. J. M. (1989). Speaking: From intention to articulation. Cambridge: The MIT Press.

MacWhinney, B. (2000). The CHILDES Project: Tools for Analyzing Talk (3era Ed., vol. II). Nueva Jersey: Lawrence Erlbaum.

McLaughlin, B. (1990). Restructuring. Applied Linguistics, 11(2), 113-128.

McKee, G., Malvern, D. \& Richards, B. (2000). Measuring vocabulary diversity using dedicated software. Literary and Linguistic Computing, 15(3), 323-337.

Norris, J. \& Ortega, L. (2009). Towards an organic approach to investigating CAF in instructed SLA: The case of complexity. Applied Linguistics, 30(4), 555-578.

Pawlak, M. (2011). Instructed acquisition of speaking: Reconciling theory and practice. En M. Pawlak, E. Waniek-Klimczak \& J. Maje (Eds.), Speaking and instructed foreign language acquisition (pp. 3-19). Bristol Buffalo, Toronto: Multilingual Matters.

Pienemann, M. (1998). Language processing and second language development: Processability theory. Philadelphia: John Benjamins. 
Pienemann, M. \& Lenzing, A. (2015). Processability theory. En B. VanPatten \& J. Williams (Eds.), Theories in Second Language Acquisition: An Introduction (pp. 159179). NuevaYork: Routledge.

Robinson, P. (2011). Second language task complexity, the cognition hypothesis, language learning and performance. En P. Robinson (Ed.), Second language task complexity: Researching the cognition hypothesis of language learning and performance (pp. 203-236). Amsterdam: John Benjamins.

Schmidt, R. (1992). Psychological mechanisms underlying second language fluency. Studies in Second Language Acquisition, 14(4), 357-385.

Skehan, P. (1998). A cognitive approach to language learning. Oxford: Oxford University Press.

Skehan, P. (2009). Modelling second language performance: Integrating complexity, accuracy, fluency, and lexis. Applied Linguistics, 30(4), 510-532.

Skehan, P. \& Foster, P. (1997). Task type and task processing conditions as influences on foreign language performance. Language teaching research, 1(3), 185-211.

Ullman, M. (2015). The declarative/procedual model. En B. VanPatten \& J. Williams (Eds.), Theories in Second Language Acquisition: An Introduction (pp. 135-158). Nueva York: Routledge.

Vercellotti, M. (2012). Complexity, accuracy, and fluency as properties of language performance: The development of the multiple subsystems over time and in relation to each other. Tesis doctoral, Universidad de Pittsburg, Estados Unidos.

Vercellotti, M. (2015). The development of complexity, accuracy, and fluency in second language performance: A longitudinal study. Applied Linguistics, 36(2), $1-23$.

Verspoor, M. \& Van Dijk, M. (2014). Variability in a dynamic systems approach. En C. Chapelle (Ed.), The encyclopedia of Applied Linguistics (pp. 6051-6059). Oxford: Wiley-Blackwell. 